\title{
reviews
}

\section{Nuclear phenomena and development}

A. T. Sumner

Nuclear Cytology in Relation to Development. By F. d'Amato. Pp. viii +283 . (Cambridge University: New York, London and Cambridge, 1977.) $£ 15$.

THE illustration on the jacket of this book of Balbiani rings on a polytene chromosome seems particularly appropriate for the subject of Nuclear Cytology in Relation to Development. In fact, Professor d'Amato takes his subject matter from a much wider field than this illustration might suggest. "Nuclear cytology" is regarded as ranging from molecular biology to cytogenetics; what one might regard as nuclear cytology in the strict sensethe detailed study of nuclear structure -does not occupy an important place in this book. Similarly, the subject of "development" is broadly interpreted. Evidently, although this is not stated in the preface, Professor d'Amato's aim has been to describe those nuclear phenomena which are associated with the many aspects of development. A refreshing and often illuminating aspect of his treatment of the subject is the balance of examples from both the plant and animal kingdoms.

The first chapter deals with lifecycles, especially the alternation of diploid and haploid phases, and certain aspects of fertilisation. Chapter 2, on the cell cycle, opens with a brief excursion into molecular biology, discussing DNA sequence complexity, as well as the different classes of RNA. It is disappointing to find that a book published in 1977 should regard histones as "general repressors of gene activity", while making no mention of their important structural role in nucleosomes. This section seems rather oddly placed at the beginning of a chapter very much concerned with factors influencing the duration of different stages of the cell cycle, although also treating various aspects of chromosome replication. From the cell cycle, d'Amato turns to a well integrated account of meiosis, with special consideration of meiosis in relation to parthenogenesis.

It is not at all clear why chapter 4, on "Mosaics and Chimaeras" was included. Although the subject is of great relevance to several problems of development, no special nuclear phenomena are described here, apart from a short mention of chromatin elimination in cases of gonadosomic mosaicism. This chapter highlights a problem which recurs to a varying extent throughout the book. There are many excellent and well described examples in every chapter, but insufficient attempt has been made to explain their relevance to the chapter in which they occur, or to the book as a whole. Since, as already noted, the author does not clearly state his aim in writing the book, it is particularly important that a common theme should be evident throughout the book. Such a theme eventually appears as one reads the book, but there are places where it seems that the author's enthusiasm for certain topics has led to considerable digressions.

The remaining half of this book sticks more closely to what seems to be its main theme: nuclear phenomena correlated with development. Two chapters deal with changes in nuclear DNA content during development: one with increases of the whole chromosome complement (essentially polyploidy and polyteny), and the other with differential DNA replication. There is much of interest in both these chapters, and the author's practice of taking examples from a wide variety of organisms is of particular advantage here. Evidently, DNA amplification, either selective or total, may be more important in differentiation than I. for one, had supposed.

Next come two chapters which cover what one might regard as the essence of a study of the nucleus in relation to development: gene expression and its regulation. It is axiomatic, as d'Amato clearly states, that differentiation must be viewed in terms of gene expression. Although none of the nuclear changes described earlier in this book can be causally related to differentiation, it is clear that single somatic nuclei of plants and (as described in the final chapter) of animals contain all the genetic information necessary for normal development. d'Amato's consideration of gene expression concentrates on the localisation by in situ hybridisation of repeated genes, and on transcription in lampbrush and polytene chromosomes. These latter aspects could perhaps have been treated more extensively with advantage, for here are two types of chromosomes which afford unrivalled material for the study of nuclear cytology in relation to development. It is, moreover, a section which calls for half-tone illustrations, of which there are none in the book. If these were rejected on grounds of expense, it seems a false economy. (The book is nevertheless well illustrated by line drawings.) In the following chapters, the roles of heterochromatin, histones, and non-histone chromosomal proteins in gene regulation are considered, with the growing evidence for the Importance of the latter being clearly brought out. The final chapter, "Regeneration and Totipotency," brings together information interesting in its own right, including the exciting experiments on nuclear transplantation in amphibia. This work, showing that nuclei of differentiated somatic cells still contain all the information necessary to produce a complete adult organism, is now such a cornerstone of developmental biology that it deserves an earlier and more prominent place in this book.

In conclusion, Professor d'Amato has brought together a wealth of information on the changes which occur in cell nuclei in different developmental situations. This is reflected in a bibliography of (the publisher tells us) over 1100 references, occupying nearly 50 pages. One wishes that the author had taken more space for general comment to integrate his material Nevertheless, this is a book in which the specialist may well see new light shining on old problems, and the non-specialist read profitably for general information.

A. T. Sumner is a member of the scientific staff of the MRC Clinical and Population Cytogenetics Unit, Edinburgh, UK. 